to original work and for further study are provided at the end of each chapter. The series title International tables for crystallography for Volume D is justified thanks to the combination of many useful tables with comprehensive explanatory parts and due to setting standards of the basic concepts in this field for the international community. It fills a gap in that it provides an up-to-date reference enriched by useful software. The book addresses tools and subjects that are of special interest to researcher and postgraduate students who want a better quantitative understanding of known materials and the development of new materials. This may concern not only the traditional readers of International tables for crystallography, but also solid-state physicists, chemists and materials scientists. Thus, people who want to be guided in their study or research by a sound and comprehensive reference to crystal physics should have access to ITD.

Finally, special thanks are expressed to the editor, André Authier, to all contributing authors, and to the Technical editors, Nicola Ashcroft and Amanda Berry, for their tremendous work.

\section{P. Paufler}

Department of Physics

Dresden University of Technology

D-01062 Dresden

Germany

E-mail: paufler@tu-dresden.de

\section{books received}

The following books have been received by the Editor. Brief and generally uncritical notices are given of works of marginal crystallographic interest; occasionally, a book of fundamental interest is included under this heading because of difficulty in finding a suitable reviewer without great delay.

Cristallographie. Deuxième édition revue et augmentée. Par Dieter Schwarzenbach et Gervais Chapuis. Paris: Presses Polytechniques et Universitaires Romandes, 2006. Pp. xii +344 . Prix broché EUR 55.45. ISBN 2-88074-672-8.

Utilisant des connaissances d'algèbre linéaire et la transformation de Fourier, la présentation de la matière est néanmoins essentiellement géométrique. La nécessité de se familiariser avec la vision tridimensionelle d'objets étant probablement la difficulté la plus importante rencontrée par les étudiants, un soin particulier a été apporté à la réalization des figures. Ce livre comporte en outre toute une série d'exercices et leur solution. Sommaire: 1. Cristallographie géométrique, 2. Symétrie, 3. Diffraction des rayons X par les cristaux,

4. Résolution de structures cristallines,

5. Éléments de structures cristallines,

6. Propriétés tensorielles des cristaux, 7. Exercices.
Franz Ernst Neumann (1798-1895). Zum 200. Geburtstag des Mathematikers, Physikers und Kristallographen. Herausgeber R. Fritsch, E. Neumann-Redlin-von Neumann und T. J. Schenck. (Bilingual German/Russian.) Kaliningrad und München: Terra Baltica und LudwigMaximilians-Universität, 2005. 252 Seiten. Broschiert Preis: EUR 10. ISBN 598777-005-X, 3-922480-17-9.

Aus Anlass des 200. Geburtstages von Franz Neumann fand 1998 sowohl ein Seminar im Deutsch-Russischen Haus von Kaliningrad (früher: Königsberg) als auch ein Dies Academicus an der LMU München statt. Der vorliegende Band enthält die Ansprachen und wissenschaftlichen Beiträge in deutscher und russischer Fassung. Inhalt: K. K. Lavrinovič: Eröffnung; E. NeumannRedlin-von Meding: Franz Ernst Neumann (11.9.1798-23.5.1895); E. F. Kondrat'ev: Franz Ernst Neumann - ein Vektor, in die Zukunft weisend; P. Paufler: Tensoreigenschaften trikliner Kristalle: Eine Hommage auf Franz Ernst Neumann; J. Batt: Die Bedeutung Franz Ernst Neumanns für die Mathematik; K. K.Lavrinovič: F. E. Neumann und F. W. Bessel; P. Roquette: Königsberger Mathematiker im 19. Jahrhundert; R. Fritsch: Mathematiker unter Franz Ernst Neumanns Nachkommen; O. M. Badmaeva: Plastische Portraits von Franz Ernst Neumann. Anhang. 